\title{
LIMITING BEHAVIOR OF A CLASS OF NONLINEAR REACTION DIFFUSION EQUATIONS*
}

\author{
BY CHARLES J. HOLLAND (Office of Naval Research, Arlington, Va.)
}

1. Introduction. In this paper we determine the precise rate of decay to the zero solution of solutions $u(t, x)$ to the semilinear parabolic equation

$$
u_{t}=\Delta u+a(x) u+g(u)
$$

in a smooth bounded domain $S$ with zero lateral data $u=0$ on $t>0 \times \partial S$ and sufficiently small initial data $u(0, x)=M(x)$ for $x \in S$. Here $g(0)=g^{\prime}(0)=0$, and hence $g$ has no linear contribution.

We show in Theorem 1 that if $M$ is sufficiently small, then $u e^{\lambda t} \rightarrow C^{*} \phi$ as $t \rightarrow \infty$ in $H_{0}^{1}(S)$ for some constant $C^{*}$ (positive, negative, or zero) where $-\lambda$, assumed negative, is the principal eigenvalue to the operator

$$
L v=\Delta v+a(x) v
$$

with zero Dirichlet boundary conditions and $\phi$ is the corresponding $L_{2}(S)$ normalized non-negative eigenfunction.

Thus Theorem 1 justifies the result that is expected from a formal application of linearization. Moreover, the smallness assumption on the initial data can be relaxed using a recent result of Lions [6]. Lions has shown that if $a=0, g^{\prime \prime}>0$, and if $K$ denotes the set of initial data for which $|u(t, x)|<c$ for some constant $c$ independent of $(t, x)$, then $K$ is an unbounded convex set and $u \rightarrow 0$ as $t \rightarrow \infty$ in $C^{2}(\bar{S})$ for any initial data in the interior of $K$. Theorem 1 can then be applied to deduce that $u e^{\lambda t} \rightarrow C^{*} \phi$ as $t \rightarrow \infty$ for any initial data in the interior of $K$ without the restriction of small initial data (see Corollary 1).

Our method of proof in Theorem 1 utilizes the well-known method of Liapunov (energy) functionals (see [2], [3], [4]) with two modifications. First, it is necessary to derive a weak a priori bound on the solution $u$ (see Lemma 1) that requires the use of the maximum principle for parabolic equations. Second, we are unable to show that the energy $E(t)$ is decreasing along solutions to (1), but are able only to establish that $E^{\prime}(t)=-d_{1}(t)+d_{2}(t)$ where $d_{1}$ is non-negative and $d_{2}$ is absolutely integrable on $(0, \infty)$. However, this estimate on $E^{\prime}$ is sufficient to derive the result with use of the minimizing property of the function $\phi$.

2. A preparatory lemma. Throughout we shall assume the following:

(A) The function $g$ is of class $C^{2}(R)$ and $g(0)=g^{\prime}(0)=0$.

(B) The principal eigenvalue to the linear operator $L$ defined in (2) is negative.

(C) The initial data $u(0, x)$ is of class $C^{1}(S)$ with $u(t, x)=0$ for $x$ on the boundary of $S$.

Define, for each $r>0, U^{*}(r)$ as the infimum of $|u|$ such that $g(u)=r u$. Since (A) holds, $U^{*}(r)$ is positive for any positive $r$. We allow the possibility that $U^{*}(r)$ may be $+\infty$. Note

* Received September 25, 1981. 
that $g(u)<r u$ for $0<u<U^{*}(r)$ and $g(u)>r u$ for $-U^{*}(r)<u<0$. The conclusions of the following lemma are required in the proof of the theorem.

Lemma 1. Assume (A), (B), (C). Suppose there exist constants $C, r$ with $0<r<\lambda$ such that

$$
|u(0, x)|<C \phi(x)<U^{*}(r) .
$$

Then the solution to (1) exists for all time $t>0$ and satisfies

$$
|u(t, x)|<C \phi(x) \exp (-\lambda+r) t .
$$

Proof. Let $u^{+}\left(u^{-}\right)$be the solution to (1) with initial data $u^{+}(x, 0)=\max (u(x, 0), 0)$ $\left(u^{-}(x, 0)=\min (u(x, 0), 0)\right)$. With the use of comparison theorems (see [5], p. 125 for a recent reference), one obtains that

$$
u^{-} \leq u \leq u^{+}, \quad u^{-} \leq 0 \leq u^{+} .
$$

We first obtain the lower bound implied by (3) for $u^{-}$and hence for $u$. Let $t_{1}$ be the first time $t>0$ such that $u^{-}(t, x)=U^{*}(r)$ for some $x$ in $S$ and let $v(t, x)=-C \phi(x) \exp (-\lambda+r) t$. Now on $\left(0, t_{1}\right) \times S, v$ satisfies

$$
v_{t}=L v+r v
$$

While $u^{-}$satisfies

$$
u_{t}^{-} \geq L u^{-}+r u^{-}
$$

since $g(u) \geq r u$ if $U^{*}(r) \leq u \leq 0$. Since initially $v \leq u^{-}$and $v=u^{-}=0$ on the lateral boundary, comparison theorems yield that $v(t, x) \leq u^{-}(t, x)$ on $\left(0, t_{1}\right) \times S$. Thus $t_{1}=\infty$, and the lower bound is established.

The upper bound is established in a similar manner. The details are omitted.

3. The theorem. We are now ready to prove the main theorem.

THEOREM 1. Assume that (3) holds with $\lambda>3 r$. Then there exists a constant $C^{*}$ such that

$$
u(t, \cdot) \exp \lambda t \rightarrow C^{*} \phi(\cdot) \quad \text { as } \quad t \rightarrow \infty \quad \text { in } \quad H_{0}^{1}(S) \text {. }
$$

Proof: Since $u$ satisfies the decay bound (4) and $g(0)=g^{\prime}(0)=0$, then we may assume that $|g(u)| \leq C_{1} u^{2}$ and $\left|u g^{\prime}(u)\right| \leq C_{1} u^{2}$ for all $u$ with $C_{1}=\max \left|g^{\prime \prime}(u)\right|$ for $|u| \leq U^{*}(r)$.

Define $v(t, x)=u(t, x) \exp \lambda t$ and

$$
E(t)=\int_{S}\left[\left|v_{x}(t, x)\right|^{2}-(a(x)+\lambda)(v(t, x))^{2}\right] / 2-e^{\lambda t} G(t, v(t, x)) d x
$$

with $G(t, v)=\int_{0}^{v} g\left(p e^{-\lambda t}\right) d p$.

We first show that $E(t)$ is bounded below on $(0, \infty)$. Note that Lemma 1 establishes that

$$
\int_{S}|v(t, x)|^{a} d x \leq C(q) \exp g r t
$$

for any positive integer $q$. We first estimate the term

$$
\left|\int(\exp \lambda t) G(t, v(t, x)) d x\right| \text {. }
$$

Utilizing the above bounds on $g(u)$ and (10), one obtains that the term (11) is bounded above by $K_{1} \exp (3 r-\lambda) t$ for some constant $K_{1}$. Hence the term (11) has limit 0 as $t \rightarrow \infty$. 
We next establish that $\int_{s} v^{2}(t, x) d x$ is bounded. A direct calculation shows that $v$ satisfies the equation

$$
v_{t}=\Delta v+a(x) v+\lambda v+\exp \lambda t g(v \exp -\lambda t)
$$

and $v(t, x)=0$ for $x \in \partial S$. Hence $\int v^{2}(t, x) d x$ satisfies

$$
(1 / 2) \frac{d}{d t} \int v^{2} d x=\int_{s}-\left|v_{x}\right|^{2}+(a(x)+\lambda) v^{2}+e^{\lambda t} v g\left(v e^{-\lambda t}\right) d x
$$

with $v$ evaluated at $(t, x)$. Using the Rayleigh-Ritz characterization of the principal eigenvalue $\lambda$ and the bound on $g(u)$, one obtains that the right-hand side is bounded above by

and hence

$$
e^{2 \lambda t} \int_{S} e^{-\lambda t} v g\left(v e^{-\lambda t}\right) d x
$$

$$
(1 / 2) \frac{d}{d t} \int v^{2} d x \leq e^{2 \lambda t}\left(k_{3} \exp 3(r-\lambda) t\right)
$$

for an appropriate constant $k_{3}$. Integrating the differential inequality (13), one obtains that

for any $t>0$.

$$
\int_{S} v^{2}(t, x) d x-\int_{S} v^{2}(0, x) d x \leq \text { constant }
$$

Using the two estimates derived above, one obtains that $E(t)$ is bounded below. Moreover, the inequality (13) guarantees that $\int v^{2}(t, x) d x$ approaches a limit as $t \rightarrow \infty$. We shall need this additional fact later.

We next compute $E^{\prime}(t)$. Differentiating and using the equation (12) for $v$, one obtains that

$$
\begin{aligned}
E^{\prime}(t)= & \int_{S}-v_{t}^{2}(t, x) d s-\lambda e^{\lambda t} \int_{S} G(t, v(t, x)) d x \\
& +\lambda e^{\lambda t} \int_{S} g^{\prime}\left(p e^{-\lambda t}\right) p e^{-\lambda t} d x .
\end{aligned}
$$

Using the bounds above on $g$ and $g^{\prime}$, one obtains that

for some positive constant $k_{5}$.

$$
E^{\prime}(t) \leq-\int_{S} v_{t}^{2}(t, x) d x+k_{5} \exp (3 r-\lambda) t
$$

Since $E(t)$ is bounded below, from (15) we obtain that there exists a sequence of times $t_{n} \rightarrow \infty$ such that $\int v_{t}^{2}(t, x) d x \rightarrow 0$ as $t_{n} \rightarrow \infty$.

Utilizing the estimates derived above, one easily shows that $\int_{s}\left|\Delta v\left(t_{n}, x\right)\right|^{2} d x$ is bounded for this sequence of times. Since $v$ satisfies (12), there is a subsequence, again denoted by $t_{n}$, such that $v\left(t_{n}, \cdot\right) \rightarrow R(\cdot)$ in $H_{0}^{1}(S)$ for some function $R$ (see [1], p. 46.).

Next we show that $R$ is a solution to $L R=-\lambda R$ with $R=0$ on $\partial S$. To do this multiply (12) by any p $\in C^{\infty}(S)$ with compact support in $S$ and integrate over $S$. Integrating one term by parts and letting $t_{n} \rightarrow \infty$, one obtains that

$$
\int_{S} \nabla R \nabla p-(a(x)+\lambda) R p d x=0 .
$$


Since (16) holds for all $p$ in $C^{\infty}(S)$ with compact support and $R=0$ on $\partial S$, then standard results yield that $R$ is a classical solution to the linear equation $L R=-\lambda R$, and hence $R=C^{*} \phi$ for some constant $C^{*}$.

To complete the proof, we must show that $v(t, \cdot) \rightarrow C^{*} \phi(\cdot)$ in $H_{0}^{1}(S)$ as $t \rightarrow \infty$ and not just for the above subsequence. Recall that we may conclude from (13) that $\int_{S} v^{2} d x \rightarrow$ $\int_{S}\left(C^{*} \phi\right)^{2} d x$ as $t \rightarrow \infty$. Moreover, we know that $C^{*} \phi$ is the unique minimizer of the functional

$$
K(p)=\int_{S}|\nabla p|^{2}-a(x) p^{2} d x .
$$

subject to the restriction $\int p^{2}=\left(C^{*}\right)^{2}$.

Now, from the estimate (15) on $E^{\prime}$, we may conclude that $E(t)$ is bounded for all time. Utilizing the bounds (11) and (14), one obtains that $v(t, \cdot)$ is bounded in $H_{0}^{1}(S)$. Thus for any sequence $t_{n} \rightarrow \infty$ as $n \rightarrow \infty$, there exists a subsequence, again denoted by $t_{n}$, such that $v(t, \cdot) \rightarrow Z(\cdot)$ weakly in $H_{0}^{1}(S)$ and strongly in $L_{2}(S)$ for some function $Z$ in $H_{0}^{1}(S)$.

Since $\int|\nabla p|^{2} d x$ is lower-semicontinuous with respect to weak convergence, we can then conclude that $K(Z) \leq K\left(C^{*}\right)$ and $\int Z^{2}=\left(C^{*}\right)^{2}$. Utilizing the minimizing property (17) of $C^{*} \phi$, we may conclude that $Z=C^{*} \phi$. Since every sequences of times has a subsequence for which $v\left(t_{n}, \cdot\right) \rightarrow C^{*} \phi(\cdot)$ in $H_{0}^{1}(S), v(t, \cdot) \rightarrow C^{*} \phi(\cdot)$ in $H_{0}^{1}(S)$ as $t \rightarrow \infty$. This completes the proof of the theorem.

4. Extensions to "larger" initial data. Recall the definition of $K$ in the introduction as the set of initial data for which the absolute value of the solution to (1) is uniformly bounded for positive time. Then we record for completeness the following result.

Corollary 1. Suppose $u(0, x)$ is in the interior of $K, a=0, g^{\prime \prime}$ is positive, and assumptions (A), (B), (C) hold. Then (8) holds for some constant $C$.

Proof. From Lions [6], we have that $u(t, x) \rightarrow 0$ as $t \rightarrow \infty$ in $C^{2}(\bar{S})$. Since $|\partial \phi / \partial n|>0$, as follows from the Hopf maximum principle, then there exists $T^{*}$ such that $u\left(x, T^{*}\right)$ used an initial data for (1) would satisfy the smallness hypothesis of Theorem 1. Let $z$ denote the solution to (1) with initial data $z(0, x)=u\left(T^{*}, x\right)$. Since for $t>T^{*}$,

$$
u(t, x) \exp \lambda t=\left(z\left(t-T^{*}, x\right) / \exp -\lambda\left(t-T^{*}\right)\right) \exp \lambda T^{*},
$$

an application of Theorem 1 to the function $z$ yields the desired result.

\section{REFERENCES}

[1] M. Berger, Nonlinearity and functional analysis, New York and London, Academic Press, 1977

[2] N. Chafee, $A$ stability analysis for a semilinear parabolic partial differential equation, J. Diff. Eqns. 15, 522-540 (1974)

[3] N. Chafee and E. Infante, A bifurcation problem for a nonlinear partial differential equation of parabolic type, Applicable Anal. 4, 17-37 (1974)

[4] C. Dafermos, Asymptotic behavior of solutions to evolution equations, in Nonlinear evolution equations, M. Crandall, ed., New York and London: Academic Press, 1978, pp. 109-123

[5] P. Fife, Mathematical aspects of reacting and diffusing systems, New York, Springer-Verlag, 1979

[6] P. Lions, Asymptotic behavior of some nonlinear heat equations, MRC Technical Report \# 2134(1980) 